Comment. Math. Helv. 74 (1999) 657-670

(C) 1999 Birkhäuser Verlag, Basel

$0010-2571 / 99 / 040657-13 \$ 1.50+0.20 / 0$

Commentarii Mathematici Helvetici

\title{
The finiteness obstruction for loop spaces
}

\author{
D. Notbohm
}

\begin{abstract}
For finitely dominated spaces, Wall constructed a finiteness obstruction, which decides whether a space is equivalent to a finite $C W$-complex or not. It was conjectured that this finiteness obstruction always vanishes for quasi finite $H$-spaces, that are $H$-spaces whose homology looks like the homology of a finite $C W$-complex. In this paper we prove this conjecture for loop spaces. In particular, this shows that every quasi finite loop space is actually homotopy equivalent to a finite $C W$-complex.
\end{abstract}

Mathematics Subject Classification (1991). 57Q12, 55R35, 55R10

Keywords. Finiteness obstruction, Wall obstruction, loop space, p-compact group

\section{Introduction}

A topological space is called quasi-finite, if the direct sum $\bigoplus_{n} H_{n}(X ; \mathbb{Z})$ of all integral homology groups is a finitely generated abelian group. Then you can ask whether the space $X$ is (weakly) homotopy equivalent to a finite $C W$-complex. For finitely dominated spaces, Wall constructed an invariant which decides this problem [29] [30]. A space $X$ is finitely dominated, if there exist a finite $C W$ complex $K$ and maps $f: K \rightarrow X$ and $g: X \rightarrow K$ such that the composition $f g$ is homotopic to the identity $i d_{X}$ on $X$. One can show that finitely dominated spaces are always homotopy equivalent to $C W$-complexes and that each component is path connected (e.g. see [17, Section 2]). Examples of finitely dominated spaces are given by quasi-finite nilpotent spaces. Actually, for nilpotent space, both conditions, finitely dominated and quasi-finite are equivalent [15]. A space $X$ is called nilpotent if $X$ is homotopy equivalent to a $C W$-complex and if $\pi_{1}(X)$ acts nilpotent on the homotopy groups $\pi_{*}(X)$. In particular, $\pi_{1}(X)$ is a nilpotent group. The space $X$ is called simple if, in addition, the action is trivial. In this case, $\pi_{1}(X)$ is abelian.

For a finitely dominated space $X$, the finiteness obstruction $\tilde{\omega}(X)$ of Wall is an element in the reduced projective class group $\tilde{K}_{0}\left(\mathbb{Z}\left[\pi_{1}(X)\right]\right)$ of the integral group ring. And $\tilde{\omega}(X)$ vanishes if and only if $X$ is homotopy equivalent to a finite $C W$-complex [29] [30]. It is interesting to investigate the question how topological 
properties of spaces are reflected by properties of the finiteness obstruction. For instance, it is not known, but conjectured, that the finiteness obstruction for quasifinite loop spaces or for $H$-spaces always vanishes. In this paper we will prove this conjecture for loop spaces.

A loop space $L$ consists of a triple $(L, B L, e)$, where $L$ and $B L$ are of the homotopy type of a $C W$-complex, $B L$ pointed, and where $e: \Omega B L \rightarrow L$ is a homotopy equivalence between the loop space of $B L$ and $L$. Examples of loop spaces are given by topological groups and their classifying spaces. Loop spaces inherit properties from the space $L$; e.g. $L$ is called quasi-finite, if the space $L$ is so. Loop spaces are always nilpotent spaces. In fact, they are simple. Thus every quasi-finite loop space is finitely dominated and the finiteness obstruction $\tilde{\omega}(L)$ is defined.

Theorem 1.1. Let $L$ be a quasi finite loop space. Then $0=\tilde{\omega}(L) \in \tilde{K}_{0}\left(\mathbb{Z}\left[\pi_{1}\right]\right)$ and $L$ is homotopy equivalent to a finite $C W$-complex.

For a quasi-finite nilpotent space $X$, Mislin proved results of this type if $\pi_{1}(X)$ is infinite [16] or if $\pi_{1}(X)$ is cyclic of prime power order [15].

The proof of our result is a consequence of the next two statements. For every fibration $F \rightarrow E \rightarrow B$, the fiber transport along loops in $B$ establishes a homomorphism $\pi_{1}(B) \rightarrow[F, F]$ from the fundamental group of $B$ into the set of homotopy classes of self maps of the fiber $F[31$, p. $98 \mathrm{ff}]$. The fibration is called oriented if this homomorphism is trivial.

Theorem 1.2. Let $L$ be a quasi-finite loop space, such that $\pi_{1}(L)$ is finite. Let $r:=\operatorname{dim}_{\mathbb{Q}} H^{4}(B L, \mathbb{Q})$. Then there exist a semi simple connected compact Lie group $G$ and an oriented fibration

$$
G \rightarrow L \rightarrow E
$$

such that the following holds:

(1) For the universal cover $\tilde{G}$ of $G$, we have $\tilde{G} \cong S U(2)^{r}$.

(2) The space $E$ is simple and quasi-finite.

Theorem 1.3. Let $X$ be a finitely dominated space, such that $\pi_{1}(X)$ is finite. If, up to homotopy, there exists an oriented fibration

$$
G \rightarrow X \rightarrow B
$$

such that $G \neq\{*\}$ is a connected compact Lie group and $B$ a finitely dominated space, then, the finiteness obstruction $\tilde{\omega}(X)$ vanishes and $X$ is homotopy equivalent to a finite complex.

For principal $G$-fibrations $X \rightarrow B$ Theorem 1.3 is proved in [22]. In the general case, the statement is known and a simple consequence of some vanishing results 
of Lück for the algebraic transfer in algebraic $K$-theory associated to a fibration [14] (see Section 2). We couldn't find a reference for it, but believe that it is worth to be stated.

Proof of Theorem 1.1. If $\pi_{1}(L)$ is infinite, we can apply the above mentioned result of Mislin [16]. If $\pi(L)$ is finite, the statement follows from Theorem 1.2 and Theorem 1.3, since every quasi finite nilpotent space is finitely dominated [16].

Theorem 1.2 is a corollary or a weak version of the next statement.

Theorem 1.4. Let $L$ be a quasi-finite loop space, such that $\pi_{1}(L)$ is finite. Let $r:=\operatorname{dim}_{\mathbb{Q}} H^{4}(B L, \mathbb{Q})$. Then there exist a semi simple connected compact Lie group $G$, quasi-finite loop spaces $M$ and $N$ and a fibration

$$
E \rightarrow B M \rightarrow B N
$$

such that the following holds:

(1) For the universal cover $\tilde{G}$ of $G$, we have $\tilde{G} \cong S U(2)^{r}$.

(2) The spaces $G$ and $M$ are homotopy equivalent as well as $L$ and $N$.

(3) The space $E$ is simple and quasi-finite.

The proof of Theorem 1.4 goes as follows. Since completion turns the quasifinite loop space $L$ into a $p$-compact $\operatorname{group}\left(L_{p}^{\wedge}, B L_{p}^{\wedge}, e_{p}^{\wedge}\right)$ we can use the theory of $p$-compact groups to construct the above fibration at each prime (see Section 3 ). Localized at 0 , the existence of the fibration is basically a consequence of the fact that the rationalization $B L_{0}$ is equivalent to a product of rational EilenbergMacLane spaces. Using the arithmetic square we glue all these data together and get the fibration claimed in Theorem 1.4.

In Section 2, we prove Theorem 1.3. The other sections are devoted to the proof of Theorem 1.4. In Section 3, we prove the $p$-completed version of Theorem 1.4. In Section 4 we recall some material about the arithmetic square and the genus of a space. Section 5 contains the construction of the fibration of Theorem 1.4. The analysis of the genus of those compact Lie groups appearing in Theorem 1.4 is worked out in section 6 and will complete the proof of Theorem 1.4.

We will switch between the $p$-adic completion of Bousfield and Kan [2] and the $p$-profinite completion of Sullivan [28]. But, for nilpotent spaces with mod-p homology of finite type, both constructions coincide [2].

It is a pleasure thank Wolfgang Lück for telling me that a result like Theorem 1.2 implies the vanishing of the finiteness obstruction for quasi finite loop spaces and for several helpful discussions on this subject. I am also grateful to the SFB 478 "Geometrische Strukturen in der Mathematik" at Münster for its hospitality when part of this work was done. 


\section{Proof of Theorem 1.3}

For a fibration $F \stackrel{i}{\rightarrow} E \stackrel{p}{\rightarrow} B$ with finitely dominated total space, base and fiber, Ehrlich [11] constructed a geometric transfer

$$
p^{!}: K_{0}\left(\mathbb { Z } [ \pi _ { 1 } ( B ) ] \rightarrow K _ { 0 } \left(\mathbb{Z}\left[\pi_{1}(E)\right]\right.\right.
$$

for the projective class groups of the integral group rings of the fundamental groups (see also [25]). The map $p^{\text {! }}$ also gives a transfer for the reduced projective class groups. Ehrlich also proved a formula relating the finiteness obstruction of $E$ and $B[12]$, namely

$$
\tilde{\omega}(E)=p^{!}(\tilde{\omega}(B))+\chi(B) \cdot i_{*}(\tilde{\omega}(F)) \in \tilde{K}_{0}\left(\mathbb{Z}\left[\pi_{1}(E)\right]\right),
$$

where $\chi(B)$ denotes the Euler characteristic of $B$ and where $i_{*}: \tilde{K}_{0}\left(\mathbb{Z}\left[\pi_{1}(F)\right] \rightarrow\right.$ $\tilde{K}_{0}\left(\mathbb{Z}\left[\pi_{1}(E)\right]\right.$ is the map induced by induction.

In [13] [14], for fibrations of the above type, Lück constructed an algebraic transfer, showed that both coincide and proved the following vanishing result.

Theorem 2.1. (Lück $[14,9.1])$ Let $G \rightarrow E \rightarrow B$ be an oriented fibration, such that $G$ is a connected compact Lie group. If $\pi_{1}(B)$ is finite, then the algebraic transfer

$$
p^{*}: K_{0}\left(\mathbb { Z } [ \pi _ { 1 } ( B ) ] \rightarrow K _ { 0 } \left(\mathbb{Z}\left[\pi_{1}(E)\right]\right.\right.
$$

vanishes.

Proof of Theorem 1.3. The compact Lie group $G$ is connected and homotopy equivalent to a finite $C W$-complex. Hence $\tilde{\omega}(G)=0$. Moreover, $\pi_{1}(B)$ is finite since $\pi_{1}(X)$ is. Thus, the statement is a simple consequence of Theorem 2.1 and the above formula.

\section{Particular subgroups of $p$-compact groups}

A $p$-compact group $X$ is a loop space $X=(X, B X, e)$ such that $B X$ is $p$-complete and pointed and such that $X$ is $\mathbb{F}_{p}$-finite, i.e. $H^{*}\left(X ; \mathbb{F}_{p}\right)$ is finite in each degree and vanishes in almost all degrees. As turned out [6] [7] [20] $p$-compact groups behave very much like compact Lie groups. In particular, most of the classical notions for compact Lie groups are available in this context; e.g. there exist subgroups, maximal tori and Weyl groups with the same properties and we can speak of centralizers of $p$-compact toral subgroups. A $p$-compact toral group $P$ is a $p$-compact group $P$ such that $\pi_{0}(P) \cong \pi_{1}(B P)$ is a finite $p$-group and such that the universal cover of $B P$ is equivalent to an Eilenberg-MacLane space of the form $K\left(\mathbb{Z}_{p}^{\wedge n}, 2\right)$. For details and further notions we refer the reader to the above mentioned references and/or to the survey articles [5], [19] and [24]. 
In this section we want to prove the following statement, which is a $p$-completed version of Theorem 1.4.

Proposition 3.1. Let $X$ be a connected p-compact group, such that $\pi_{1}(X)$ is finite. Let $r:=\operatorname{dim}_{\mathbb{Q}} H^{4}\left(B X ; \mathbb{Z}_{p}^{\wedge}\right) \otimes \mathbb{Q}$. Then there exists a connected compact Lie group $G$ and a map

$$
B G_{p}^{\wedge} \stackrel{f}{\rightarrow} B X
$$

such that the following holds:

(1) For the universal cover $\tilde{G}$ of $G$, we have $\tilde{G} \cong S U(2)^{r}$. If $p$ is odd, we can choose $G=S U(2)^{r}$.

(2) The induced map $H^{4}\left(B X ; \mathbb{Z}_{p}^{\wedge}\right) \otimes \mathbb{Q} \rightarrow H^{4}\left(B G_{p}^{\wedge} ; \mathbb{Z}_{p}^{\wedge}\right) \otimes \mathbb{Q}$ is an isomorphism.

(3) The homotopy fiber $X / G_{p}^{\wedge}$ of $f$ is simple and $\mathbb{F}_{p}$-finite.

Before we start with the proof, we draw one corollary. For a space $Y$ we denote by $Y\langle n\rangle$ the n-th Postnikov section and by $Y(n)$ the $n$-connected cover of $Y$. Hence these spaces fit into a fibration

$$
Y(n) \rightarrow Y \rightarrow Y\langle n\rangle
$$

Corollary 3.2. The composition $B G_{p}^{\wedge} \rightarrow B X \rightarrow B X\langle 4\rangle$ induces an equivalence $\left(B G_{p}^{\wedge}\right)_{0} \stackrel{\simeq}{\longrightarrow} B X\langle 4\rangle_{0}$.

Proof. Since $\pi_{1}(G)$ and $\pi_{1}(X)$ are finite and since $\tilde{G} \cong S U(2)^{r}$, both spaces, $\left(B G_{p}^{\wedge}\right)_{0}$ and $B X\langle 4\rangle_{0}$, are Eilenberg-MacLane spaces with non vanishing 4-th homotopy group. The universal coefficient theorem shows that $H^{4}\left(B X ; \mathbb{Z}_{p}^{\wedge}\right) \otimes \mathbb{Q}$ is the dual of $H_{4}\left(B X ; \mathbb{Z}_{p}^{\wedge}\right) \otimes \mathbb{Q}$ and hence, that $H_{4}\left(B G_{p}^{\wedge} ; \mathbb{Z}_{p}^{\wedge}\right) \otimes \mathbb{Q} \cong H_{4}\left(B X ; \mathbb{Z}_{p}^{\wedge}\right) \otimes \mathbb{Q}$. Moreover, since both spaces are rationally 3 -connected, the Hurewicz map induces isomorphisms $\pi_{4}\left(B X_{0}\right) \cong \pi_{4}(B X) \otimes \mathbb{Q} \cong H_{4}\left(B X ; \mathbb{Z}_{p}^{\wedge}\right) \otimes \mathbb{Q}$. The first isomorphism follows from $[2 ; \mathrm{V}, 4.1]$. Hence, the composition induces an isomorphism between the homotopy groups and is therefore an equivalence.

Proof of Proposition 3.1. First we assume that $X$ is simply connected, hence 2 -connected, and simple. In this context, simple means that the representation $\rho: W_{X} \rightarrow G l\left(L_{X} \otimes \mathbb{Q}\right)$ is irreducible. This representation is given by the action of the Weyl group $W_{X}$ on the maximal torus $T_{X}$ of $X$ respectively on the $p$-adic lattice $L_{X}:=H_{2}\left(B T_{X} ; \mathbb{Z}_{p}^{\wedge}\right)$.

If $X$ is 3 -connected, then $H^{4}\left(B X ; \mathbb{Z}_{p}^{\wedge}\right)=0$ and the statement is obviously true. If $\pi_{3}(X) \neq 0$, then $\pi_{3}(X) \otimes \mathbb{Q} \cong \mathbb{Q}_{p}^{\wedge k}$ is isomorphic to the dual of the $\mathbb{Q}_{p}^{\wedge}$ vector space $H^{4}\left(B X ; \mathbb{Z}_{p}^{\wedge}\right) \otimes \mathbb{Q} \cong\left(H^{4}\left(B T_{X} ; \mathbb{Z}_{p}^{\wedge}\right) \otimes \mathbb{Q}\right)^{W_{X}}$. The last isomorphism follows from [6]. Every element $q \in\left(H^{4}\left(B T_{X} ; \mathbb{Z}_{p}^{\wedge}\right) \otimes \mathbb{Q}\right)^{W_{X}}$ can be interpreted as a $W_{X}$-invariant quadratic form $q: L_{X} \otimes \mathbb{Q} \rightarrow \mathbb{Q}_{p}^{\wedge}$. Since $\rho$ is irreducible, every $W_{X}$-invariant quadratic form is definite and two such quadratic forms are 
linearly dependent. Thus, all the groups are isomorphic to $\mathbb{Q}_{p}^{\wedge}$, generated by a definite $W_{X}$-invariant quadratic form $q_{X}$ on $L_{X} \otimes \mathbb{Q}$. This implies that the pseudo reflection group $W_{X}$ is a honest reflection group, i.e. generated by elements of order 2 fixing a hyperplane of $L_{X} \otimes \mathbb{Q}$.

Let $s \in W_{X}$ be any reflection, and let $T:=\left(\left(T_{X}\right)^{s}\right)_{e}$ be the component of the unit of the fixed-point set of the action of $s$ on $T_{X}$. Then, the centralizer $C:=C_{X}(T) \subset X$ of $T$ is a connected subgroup of $X$ of maximal rank whose Weyl group $W_{C} \cong \mathbb{Z} / 2$ is generated by $s$. All this follows from [6] [7] [20]. The $p-$ compact group $C$ has a finite covering, which splits into a product of a torus and a simply connected $p$-compact group $C^{\prime}$, and $W_{C^{\prime}} \cong W_{C} \cong \mathbb{Z} / 2$ is of order two [20]. Since $S U(2)_{p}^{\wedge}$ is the only simply connected $p$-compact group with Weyl group isomorphic to $\mathbb{Z} / 2[9]$, we have $C^{\prime} \cong S U(2)_{p}^{\wedge}$ as $p$-compact group. This establishes a map $B S U(2)_{p}^{\wedge} \rightarrow B X$ respectively a homomorphism $S U(2)_{p}^{\wedge} \rightarrow X$ of $p$-compact groups. This might not be a monomorphism, but the kernel $K \subset S U(2)_{p}^{\wedge}$ of this homomorphism is a finite $p$-group and a central subgroup of $S U(2)_{p}^{\wedge}$, actually of $S U(2)$ in the honest sense of compact Lie groups [23]. The kernel can be divided out [23] and, doing this, yields a monomorphism $(S U(2) / K)_{p}^{\wedge} \rightarrow X$ respectively a map $B(S U(2) / K)_{p}^{\wedge} \rightarrow B X$. The last claim follows from [6]. The center $Z(S U(2) \cong \mathbb{Z} / 2$ is a 2 -group. Thus, for odd primes, $K=0$ and for $p=2$ the quotient $G:=S U(2) / K$ is a connected compact Lie group isomorphic to $S U(2)$ or $S O(3)$. In both cases, there exists a connected compact Lie group $G$ and a fibration $X / G_{p}^{\wedge} \rightarrow B G_{p}^{\wedge} \rightarrow B X$ satisfying condition (1) of the statement. The homotopy fiber $X / G_{p}^{\wedge}$ is simple since the homotopy fiber of a map between simply connected spaces is. Moreover, $X / G_{p}^{\wedge}$ is $\mathbb{F}_{p}$-finite since $G \rightarrow X$ is a monomorphism [6]. This establishes part (3).

Now we consider, the induced homomorphism

$$
\mathbb{Q}_{p}^{\wedge} \cong H^{4}\left(B X ; \mathbb{Z}_{p}^{\wedge}\right) \otimes \mathbb{Q} \rightarrow H^{4}\left(B G ; \mathbb{Z}_{p}^{\wedge}\right) \otimes \mathbb{Q} \cong \mathbb{Q}_{p}^{\wedge}
$$

By construction, the $W_{X}$-invariant quadratic form $q_{X}$ is mapped onto a $W_{G^{-}}$ invariant quadratic form $q^{\prime} \in H^{4}\left(B G ; ;_{p}^{\wedge}\right) \otimes \mathbb{Q}$. If $q^{\prime}=0$, the map $B G_{p}^{\wedge} \rightarrow B X$ induces the trivial map in reduced rational cohomology [1] and is therefore null homotopic [18]. This contradicts the fact that $X / G_{p}^{\wedge}$ is $\mathbb{F}_{p}$-finite and finishes the proof of the statement for simple simply connected $p$-compact groups.

Now let $X$ be a connected $p$-compact group, such that $\pi_{1}(X)$ is finite. Then the universal cover $\tilde{X}$ is again a $p$-compact group and establishes a map $B \tilde{X} \rightarrow$ $B X$ which induces an isomorphism $H^{*}\left(B X ; \mathbb{Z}_{p}^{\wedge}\right) \otimes \mathbb{Q} \stackrel{\cong}{\longrightarrow} H^{*}\left(B \tilde{X} ; \mathbb{Z}_{p}^{\wedge}\right) \otimes \mathbb{Q}[20]$. By [8], $\tilde{X} \cong \prod_{i} X_{i}$ splits into a finite product of simply connected simple $p$ compact groups $X_{i}$. For each $i$, we choose a connected compact Lie group $G_{i}$ and a monomorphism $G_{i p}^{\wedge} \rightarrow X_{i}$ satisfying the statement. Taking the product gives a homomorphism

$$
G_{p}^{\prime \wedge}:=\prod_{i} G_{i p}^{\wedge} \rightarrow \prod_{i} X_{i} \cong \tilde{X} \rightarrow X
$$


which might not be a monomorphism. But, analogously as above, the kernel $K$ is a central subgroup of $G^{\prime}$ (in the Lie group sense) and, by dividing out the kernel, the homomorphism can be made into a monomorphism $G_{p}^{\wedge}:=\left(G^{\prime} / K\right)_{p}^{\wedge} \rightarrow X$. Again, the center of $G^{\prime}$ is a 2 -group. Hence, for odd primes, $G \cong S U(2)^{r}$. Moreover, $G$ is a connected compact Lie group satisfying part (1) of the statement.

By construction, all the maps establish a commutative diagram

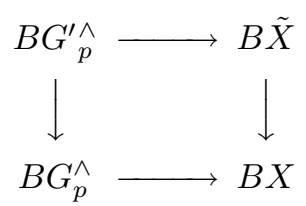

where the vertical arrows induce isomorphisms in rational cohomology. The top horizontal map satisfies part (2) and so does the bottom arrow. By the same argument as above the homotopy fiber $X / G_{p}^{\wedge}$ of the bottom horizontal arrow is simple and $\mathbb{F}_{p}$-finite.

\section{The arithmetic square and genus sets}

Most of the material in this section is taken from [28] and [32]. For a space $X$ we define $X^{\wedge}:=\prod_{p} X_{p}^{\wedge}$ to be the product of the $p$-adic completions in the sense of Bousfield and Kan [2] taken over all primes. For nilpotent spaces with mod- $p$ homology of finite type, this is equivalent to the profinite completion of Sullivan [28] [2]. Sullivan also constructed a formal completion for spaces [28]. For a space $X$, the $p$-formal completion $X_{p}^{b}$ is defined to be the homotopy colimit hocolim $X_{i} X_{p}$ where $X_{i}$ ranges over all finite subcomplexes of $X$. The formal completion $X^{b}$ is given by hocolim $_{i} X_{i}^{\wedge}$. Both completions are coaugmented functors on the homotopy category of topological spaces. That is they come with a map $X \rightarrow X_{p}^{b}$ respectively $X \rightarrow X_{p}^{\wedge}$. By $X_{0}^{b}$ we denote the formal completion of the rationalization of $X$ and by $X_{0}^{\wedge}$ the rationalization of the profinite completion of $X$.

Theorem 4.1. ([Sullivan]) Let $X$ be a connected simple $C W$-complex of finite type. Then

(1) $X_{0}^{b} \simeq X_{0}^{\wedge}$.

(2) The diagram

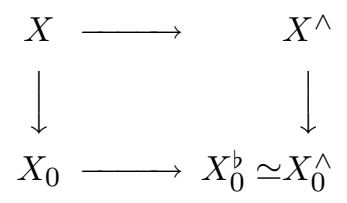

is a fiber square up to homotopy. 
Proof. Actually, in [28] this is only proved for simply connected spaces, but all proofs also work in the case of simple spaces (see also [2]).

Remark 4.2. For a simple space $X$, we can identify $X_{0}^{\wedge}$ and $X_{0}^{b}$ via the above equivalence. The homotopy groups $\pi_{*}\left(X_{0}^{\wedge}\right)$ are modules over $\mathbb{Q} \otimes \mathbb{Z}^{\wedge}$ and therefore topological groups. For simple spaces $X$ and $Y$, a map $f:\left(X^{\wedge}\right)_{0} \rightarrow\left(Y^{\wedge}\right)_{0}$ is called $\pi_{*}$-continuous if $\pi_{*}(f)$ is continuous. In the finitely generated case this is equivalent to $\pi_{*}(f)$ being $\mathbb{Q} \otimes \mathbb{Z}^{\wedge}$-linear. If $f$ is induced from a map $X_{0} \rightarrow Y_{0}$ by formal completion or from $X^{\wedge} \rightarrow Y^{\wedge}$ by localization then $f$ is always $\pi_{*^{-}}$ continuous.

If $X_{0}^{\wedge} \simeq Y_{0}^{\wedge}$ we denote by $H E C_{0}^{\wedge}(X, Y)$ the set of homotopy classes of $\pi_{*^{-}}$ continuous homotopy equivalences $f:\left(X^{\wedge}\right)_{0} \rightarrow\left(Y^{\wedge}\right)_{0}$. If $X=Y$, then we define $H E C_{0}^{\wedge}(X):=H E C_{0}^{\wedge}(X, X)$. The elements of these groups are called gluing maps.

For any element $\alpha \in H E C_{0}^{\wedge}(X, Y)$ we define $W:=W(X, \alpha, Y)$ to be the homotopy pull back of

$$
X_{0} \rightarrow\left(X^{\wedge}\right)_{0} \stackrel{\alpha}{\longrightarrow}\left(Y^{\wedge}\right)_{0} \leftarrow Y^{\wedge} .
$$

Proposition 4.3. Let $X$ and $Y$ are simple spaces of finite type and let $\alpha \in$ $H E C_{0}^{\wedge}(X, Y)$. Then, for $W:=W(X, \alpha, Y)$, the following holds:

(1) $W^{\wedge} \simeq Y^{\wedge}$ and $W_{0} \simeq X_{0}$.

(2) $W$ is a simple space of finite type.

Proof. Part (1) is obvious. By $[32 ; 3.7]$, the abelian groups $\pi_{n}(W)$ are finitely generated for all $n$ and, since $\pi_{n}(W) \rightarrow \pi_{n}\left(W^{\wedge}\right) \cong \pi_{n}\left(X^{\wedge}\right)$ is a monomorphism, $W$ is simple. This proves the second part of the statement.

If $X=Y$ is a nilpotent space of finite type, the above construction gives a map $H E C_{0}^{\wedge}(X) \rightarrow G_{0}^{\wedge}(X)$ where the genus $G_{0}^{\wedge}(X)$ of $X$ is the set of all homotopy equivalence classes of nilpotent spaces $Y$ of finite type such that $Y^{\wedge} \simeq X^{\wedge}$ and $Y_{0} \simeq X_{0}$. In $[32 ; 3.8]$ is proved that the above assignment identifies the genus set of $X$ with a particular double coset of $H E C_{0}^{\wedge}(X)$.

\section{Construction of a particular fibration}

Let $L$ be a a quasi-finite connected loop space with finite fundamental group. Then, the rational cohomology $H^{*}(B L ; \mathbb{Q})$ of the classifying space $B L$ of $L$ is a finitely generated polynomial algebra with generators $x_{1}, \ldots, x_{n}$. The generators are chosen as images of non divisible integral classes, also denoted by $x_{i} \in H^{*}(B L ; \mathbb{Z})$. These integral classes define a map

$$
B L \stackrel{e}{\rightarrow} K:=\prod_{i} K\left(\mathbb{Z},\left|x_{i}\right|\right)=: K\langle 4\rangle \times K(4)
$$


where $K\left(\mathbb{Z},\left|x_{i}\right|\right)$ is an Eilenberg-MacLane space, where $\left|x_{i}\right|$ denotes the degree of the class $x_{i}$ (which in our case is always even) and where $K\langle 4\rangle$, as the 4 -th Postnikov section, is the product of all factors of degree less than 5 and $K(4)$, as the 4-th connected cover, the product of all other factors. Rationally, this map establishes equivalences $B L_{0} \rightarrow K_{0} \simeq K\langle 4\rangle_{0} \times K(4)_{0}$ and $B L_{0}^{\wedge} \simeq B L_{0}^{b} \rightarrow K_{0}^{b} \simeq$ $K_{0}^{\wedge} \simeq K\langle 4\rangle_{0}^{\wedge} \times K(4)_{0}^{\wedge}$.

Let $r:=\operatorname{dim}_{\mathbb{Q}} H^{4}(B L ; \mathbb{Q})=\operatorname{dim}_{\mathbb{Q}_{p}^{\wedge}} H^{4}\left(B L ; \mathbb{Z}_{p}^{\wedge}\right) \otimes \mathbb{Q}$. Completed at every prime, $L$ becomes a $p$-compact group. By Proposition 3.1 and Corollary 3.2, there exist a semi simple connected compact Lie group $G$, such that $\tilde{G} \cong S U(2)^{r}$, and, for every prime $p$ a map $B G_{p}^{\wedge} \rightarrow B L_{p}^{\wedge}$ such that the composition $B G_{p}^{\wedge} \rightarrow B L_{p}^{\wedge} \rightarrow B L\langle 4\rangle_{p}^{\wedge}$ induces an equivalence after localizing at 0 . Putting all these maps together we get a map $f: B G^{\wedge} \rightarrow B L^{\wedge}$ such that $B G_{0}^{\wedge} \rightarrow B L\langle 4\rangle_{0}^{\wedge}$ is an equivalence.

Since $B G_{0}, B L\langle 4\rangle_{0}$ and $K\langle 4\rangle_{0}$ are rational Eilenberg-MacLane spaces with isomorphic homotopy groups, all these spaces are homotopy equivalent. Thus, there exists a map $s: B G_{0} \rightarrow B L_{0}$, given by a section of $B L_{0} \rightarrow K\langle 4\rangle_{0}$ such that $B G_{0} \rightarrow B L_{0} \rightarrow K(4)_{0}$ is null homotopic. We have a diagram

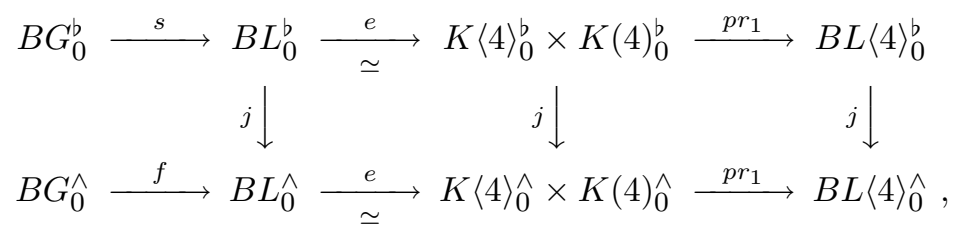

where the vertical arrows are the obvious homotopy equivalences, where $p r_{1}$ denotes the projection onto the first factor and where the composition of the horizontal arrows is a homotopy equivalence. The compositions $e s=:\left(s_{1}, s_{2}\right)$ and ef $=:\left(f_{1}, f_{2}\right)$ can be written as two components. Confusing notations, $e, f$ and $s$ as well as $f_{i}$ and $s_{i}$ also denote the maps between the uncompleted or unlocalised spaces if they exist.

We want to change the gluing map $j: B L_{0}^{b} \rightarrow B L_{0}^{\wedge}$ in such a way that it lifts to the gluing map $\alpha:=\left(p r_{1} e f\right)^{-1} p r_{1} e s: B G_{0}^{b} \rightarrow B G_{0}^{\wedge}$. This map is a homotopy equivalence and $\pi_{*}$-continuous since all homotopy groups of $B G$ and $B L$ are finitely generated and since all maps are $\mathbb{Q} \otimes \mathbb{Z}^{\wedge}$-linear. As a product of Eilenberg-MacLane spaces, $B L_{0}^{\wedge}$ is an $\mathrm{H}$-space. The product of the two maps

$$
\beta_{1}:=\left(\left.j\right|_{K\langle 4\rangle_{0}^{b}}, f_{2} \alpha\left(s_{1}\right)^{-1}\right): K\langle 4\rangle_{0}^{b} \rightarrow K\langle 4\rangle_{0}^{\wedge} \times K(4)_{0}^{\wedge}
$$

and

$$
\beta_{2}:=\left.j\right|_{K(4)_{0}^{b}}: K(4)_{0}^{b} \rightarrow K\langle 4\rangle_{0}^{\wedge} \times K(4)_{0}^{\wedge}
$$

defines a map

$$
\beta:=\beta_{1} \cdot \beta_{2}: B L_{0}^{b} \simeq K\langle 4\rangle_{0}^{b} \times K(4)_{0}^{b} \rightarrow K\langle 4\rangle_{0}^{\wedge} \times K(4)_{0}^{\wedge} \simeq B L_{0}^{\wedge}
$$


which, by construction, is $\pi_{*}$-continuous and fits into a homotopy commutative diagram

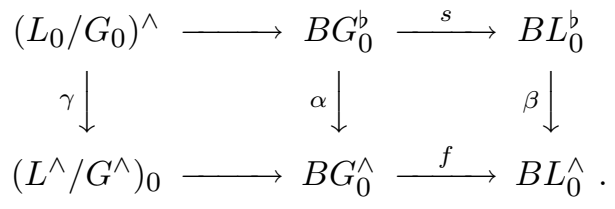

Here $L_{0} / G_{0}$ and $L^{\wedge} / G^{\wedge}$ denote the homotopy fibers of the map $s: B G_{0} \rightarrow B L_{0}$ and $f: B G^{\wedge} \rightarrow B G^{\wedge}$. The maps $\alpha$ and $\beta$ are homotopy equivalences and so is $\gamma$. Since $f$ and $s$ have left inverse, the map $\gamma$ is also $\pi_{*}$-continuous. The homotopy groups of $L_{0} / G_{0}$ are finite dimensional rational vector spaces and the homotopy groups of $L^{\wedge} / G^{\wedge}$ finitely generated $\mathbb{Z}^{\wedge}$-modules.

These considerations lead to the following proposition.

Proposition 5.1. Let $L$ be a connected quasi-finite loop space such that $\pi_{1}(L)$ is finite. Let $r:=\operatorname{dim}_{\mathbb{Q}} H^{4}(B L ; \mathbb{Q})$. Then, there exist a semi simple connected compact Lie group $G$, quasi-finite connected loop spaces $M$ and $N$ and a fibration

$$
E \rightarrow B M \rightarrow B N
$$

such that the following holds:

(1) $E$ is quasi-finite and simple.

(2) $\tilde{G} \cong S U(2)^{r}$.

(3) $B M$ is in the genus of $B G$

(4) $B N$ is in the genus of $B L$.

Proof. Using the arithmetic square, the above diagram establishes a fibration $E \rightarrow B M \rightarrow B N$. The last three properties are true by construction. The first property follows from Proposition 4.3. The loop spaces $M:=\Omega B M$ and $N:=\Omega B N$ are in the genus of $G$ respectively of $L$ and give therefore rise to quasi-finite loop spaces. This finishes the proof.

Next we want to analyze the loop $\Omega \beta: L_{0}^{b} \rightarrow L_{0}^{\wedge}$ of the gluing map $\beta$.

Lemma 5.2. $\Omega \beta \simeq j: L_{0}^{b} \rightarrow L_{0}^{\wedge}$.

Proof. Using the H-space structure of $L_{0}^{\wedge} \simeq \Omega K\langle 4\rangle_{0}^{\wedge} \times K(4)_{0}^{\wedge}$, we can again describe the map $\Omega \beta$ as a product of two maps, We just have to multiply the loops of $\beta_{1}$ and $\beta_{2}$. Therefore, we only have to show that $\left.\Omega \beta_{2}=\Omega f_{2} \alpha\left(s_{1}\right)^{-1}\right)$ : $\Omega K\langle 4\rangle_{0}^{b} \rightarrow \Omega K(4)_{0}^{\wedge}$ is null homotopic. But this map fits into a commutative diagram 


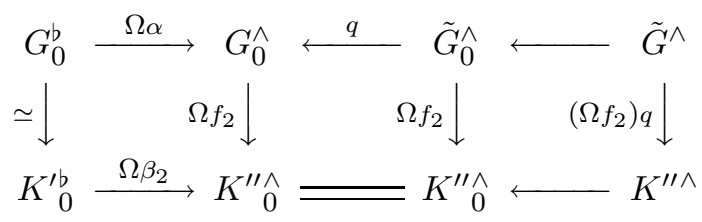

where $K^{\prime}:=\Omega K\langle 4\rangle$ and $K^{\prime \prime}:=\Omega K(4)$ are again Eilenberg-MacLane spaces. Since the first two arrows in the row are homotopy equivalences and since the map from the last column to the third column is the coaugmentation of the localization at 0 , we only have to show that $\Omega f_{2} q: \tilde{G}^{\wedge} \rightarrow K^{\prime \prime \wedge}$ is null homotopic. The EilenbergMacLane space $K(4)^{\wedge} \simeq \prod_{l} K\left(\mathbb{Z}^{\wedge}, 2 l\right)$ splits into a finite product such that $l>2$ for all $l$. Since $H^{*}\left(B \tilde{G} ; \mathbb{Z}^{\wedge}\right)$ is generated by classes of degree 4 , each coordinate of the map $B \tilde{G}^{\wedge} \rightarrow K(4)^{\wedge}$ is given by a decomposable cohomology class $x_{l}$ in $H^{*}\left(B \tilde{G}^{\wedge} ; \mathbb{Z}^{\wedge}\right)$. The next lemma finishes the proof.

Lemma 5.3. Let $L$ be a quasi-finite loop space and let $g: B L^{\wedge} \rightarrow K\left(\mathbb{Z}^{\wedge}, r\right)$ denote a decomposable cohomology class in $H^{*}\left(B L^{\wedge} ; \mathbb{Z}^{\wedge}\right)$. Then, the map $\Omega g$ : $L^{\wedge} \simeq \Omega B L^{\wedge} \rightarrow K\left(\mathbb{Z}^{\wedge}, r-1\right)$ is null homotopic.

Proof. The adjoint of $\Omega g$ factors through $\Sigma \Omega B L^{\wedge} \rightarrow B L \stackrel{g}{\rightarrow} K\left(\mathbb{Z}^{\wedge}, r\right)$. Since $\Sigma \Omega B L$ is a co-H-space, all cup products vanish. Hence, the composition is null homotopic as well as $\Omega g$.

\section{Proof of Theorem 1.4}

We start with analyzing the genus of the connected compact Lie group $G$.

Proposition 6.1. Let $G$ be a semi simple connected compact Lie group, such that $\tilde{G} \cong S U(2)^{r}$. Then, the genus of $G$ is rigid. That is every element of the genus of $G$ is actually homotopy equivalent to $G$.

Proof. First we prove the statement for $G=\tilde{G} \cong S U(2)^{r}$. Since

$G_{0}^{\wedge} \simeq K\left(\left(\mathbb{Q} \otimes \mathbb{Z}^{\wedge}\right)^{r}, 3\right)$ is an Eilenberg-MacLane space, the gluing map for $M$ is given by a matrix $A \in G l\left(r, \mathbb{Q} \otimes \mathbb{Z}^{\wedge}\right)$ and therefore splits into a product $R C$ with $R \in G l(r, \mathbb{Q})$ and $C \in G l\left(r, \mathbb{Z}^{\wedge}\right)[32]$. Since $G \cong S U(2)^{r}, R$ can be realized by a self map $G_{0} \rightarrow G_{0}$ and $C$ by a self map $G^{\wedge} \rightarrow G^{\wedge}$. This implies that $M \simeq G$ [32].

Now let $G$ be a quotient of $\tilde{G}$, and let $M \in \operatorname{genus}(G)$. Then, $\pi_{1}(M) \cong$ $\pi_{1}(G)=: E$ is a finite elementary abelian 2-group. The universal cover $M$ is contained in the genus of $\tilde{G} \simeq S U(2)^{r}$. Hence, $\tilde{M} \simeq \tilde{G}$ and $M$ fits into a fibration $\tilde{G} \rightarrow M \rightarrow B E$. The 2 -adic completion establishes an injection $\pi_{3}(\tilde{G}) \rightarrow \pi_{3}\left(\tilde{G}_{2}^{\wedge}\right)$ and turns the fibration into a principal fibration. Since principal fibration with 
connected structure groups are simple and since self maps of $\tilde{G}$ are classified by means of homotopy groups, the above fibration is oriented and is classified by a map $B E \rightarrow B S H E(\tilde{G})$, where $S H E(\tilde{G})$ denotes the set of all homotopy equivalence classes of $\tilde{G}$ homotopic to the identity. The left action of $\tilde{G}$ on itself establishes a $\operatorname{map} B \tilde{G} \rightarrow B S H E(\tilde{G})[27]$.

Now, we consider the diagram

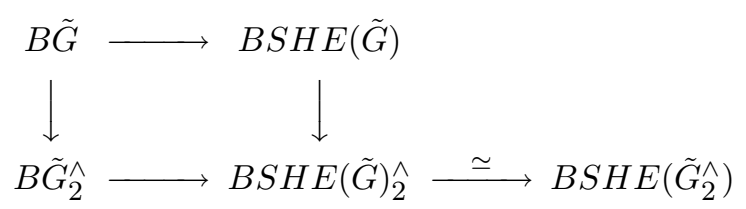

Since $\tilde{G}$ is a topological group, $S H E\left(\tilde{G}_{2}^{\wedge}\right)$ is 2-complete and the map $S H E(\tilde{G})_{2}^{1} \rightarrow$ $\operatorname{SHE}\left(\tilde{G}_{2}^{1}\right)$ is a homotopy equivalence [2]. Since completion and passing to classifying spaces commutes for nilpotent connected loop spaces [2], the second arrow in the bottom line is a homotopy equivalence. The homotopy fibers of both columns have uniquely 2-divisible homotopy groups. Thus, obstruction theory shows that for every map $B E \rightarrow B \tilde{G}_{2}^{\wedge}$ or $B E \rightarrow B S H E\left(\tilde{G}_{2}^{\wedge}\right)$ there exist a lift to $B \tilde{G}$ respectively to $B S H E(\tilde{G})$, unique up to homotopy. A small diagram chase shows that, up to homotopy, the classifying map $B E \rightarrow B S H E(\tilde{G})$ has a unique lift $B E \rightarrow B \tilde{G}$. Hence, $\tilde{G} \rightarrow M \rightarrow B E$ is a principal fibration. By [10], the classifying map is induced from a homomorphism $\rho: E \rightarrow \tilde{G}$. In fact, this map is a monomorphism, since the homotopy fiber $M$ of $B \rho$ is quasi-finite [26]. All homomorphisms $E \rightarrow \tilde{G}$ are central and therefore, $M \simeq \tilde{G} / E$ is a compact Lie group.

Finally we have to show that $\tilde{G} / E \simeq G$. Using shift maps and permutation of factors, one can show, analogously as for the equivalence $S O(4)=S U(2) \times_{\mathbb{Z} / 2}$ $S U(2) \simeq S O(3) \times S U(2)$, that $\tilde{G} / E$ and $G$ are homotopy equivalent to $S O(3)^{s} \times$ $S U^{\bullet}(2)^{r-s}$ where $s$ equals the rank of $E$.

Now we are able to finish the proof of Theorem 1.4.

Proof of Theorem 1.4. The fibration $L \rightarrow E \rightarrow B M$ of Proposition 5.1 has almost all the properties we stated in Theorem 1.4. It is only left to show that $N \simeq L$ and that $M \simeq G$. The first assertion follows from Lemma 5.2, and the second from Proposition 6.1. This finishes the proof of Theorem 1.4

\section{References}

[1] J. F. Adams \& Z. Mahmud, Maps between classifying spaces, Inventiones Math. 35 (1976), $1-41$.

[2] A. Bousfield \& D. Kan, Homotopy limits, completion and localizations, SLNM 304, Springer Verlag 1972. 
[3] W. Browder, Higher torsion in $H$-sspaces, Trans. AMS 108 (1963), 353-375.

[4] W. G. Dwyer, H. Miller, C.W. Wilkerson, The homotopy uniqueness of $B S^{3}$, Algebraic Topology Barcelona 1986, SLNM 1298, Springer, Berlin 1987, pp. 90-105.

[5] W. G. Dwyer \& C. W. Wilkerson, Homotopy fixed-point methods for Lie groups and finite loop spaces, Ann. Math. (2) 139 (1994), 395-442.

[6] W. G. Dwyer \& C. W. Wilkerson, The center of a $p$-compact group, The Čech centennial (Boston, MA, 1993), Contemp. Math. 181 (1995), 119-157.

[7] W. G. Dwyer \& C. W. Wilkerson, Product splittings of $p$-compact groups, Fund. Math. 147 (1995), 279-300.

[9] W. G. Dwyer \& C. W. Wilkerson, p-compact groups with abelian Weyl groups, Preprint.

[10] W. G. Dwyer \& A. Zabrodsky, Maps between classifying spaces. In: Algebraic Topology, Barcelona 1986, SLNM 1298, pp. 106-11.

[11] K. Ehrlich, Fibrations and transfer map in algebraic K-theory, JPPA 14 (1979), 131-136.

[12] K. Ehrlich, The obstruction to the finiteness of the total space of a fibration, Michigan Math. J. 28 (1981), 19-38.

[13] W. Lück, The transfer maps induced in the algebraic $K_{0}$ - and $K_{1}$-groups I, Math. Scand. 59 (1986) 93-121.

[14] W. Lück, The transfer map induced in the algebraic $K_{0^{-}}$and $K_{1}$-groups by a fibration II, JPPA 45 (1987) 143-169.

[15] G. Mislin, Wall's obstruction for nilpotent spaces, Topology 14 (1975), 311-317.

[16] G. Mislin, Finitely dominated nilpotent spaces, Ann. Math. 103 (1976), 547-556.

[17] G. Mislin, Wall's finiteness obstruction. In: Handbook of Algebraic topology (I. James, ed.), Elsevier 1995, pp. 1259-1292.

[18] J. M. Møller, Rational isomorphisms of $p$-compact groups, to appear.

[19] J.M. Møller, Homotopy Lie groups, Bull. Amer. Math. Soc. (N.S.) 32 (1995), 413-428.

[20] J. M. Møller \& D. Notbohm, Centers and finite coverings of finite loop spaces, J. reine u. angew. Math. 456 (1994), 99-133.

[21] J. M. Møller \& D. Notbohm, Finite loop spaces with maximal tori, Math. Gott. Heft 14 (1994).

[22] H. J. Munkholm \& E. K. Pedersen, Whitehead transfer for $S^{1}$-bundles, an algebraic description, Comm. Math. Helv. (1981), 404-430.

[23] D. Notbohm, Kernels of maps between classifying spaces, Israel J. Math. 87 (1994), 243256.

[24] D. Notbohm, Classifying spaces of compact lie groups and finite loop spaces. In: Handbook of Algebraic topology (I. James, ed.), Elsevier 1995, pp. 1049-1094.

[25] E.K. Pedersen, Universal geometric examples for transfer maps in algebraic $K$ - and $L$ theory, JPPA 22 (1981), 179-192.

[26] D. Quillen, The spectrum of an equivariant cohomology ring I, Ann. Math. 94 (1971), 549-572.

[27] J. Stasheff, A classification theorem for fiber spaces, Topology 2 (1963), 239-246.

[28] D. Sullivan, Geometric Topology, Part I: localizations, periodicity and Galois theory, Mimeographed notes, MIT 1970.

[29] C. T. C. Wall, Finiteness conditions for $C W$-complexes, Ann. Math. (2) 81 (1965), 55-69.

[30] C. T. C. Wall, Finiteness conditions for $C W$-complexes II, Proc. Roy. Soc. London Ser. A 295 (1966), 129-139.

[31] G. W. Whitehead, Elements of homotopy theory, Graduate Text in Mathematics 61, Springer, Berlin 1978.

[32] C. W. Wilkerson, Applications of minimal simplicial groups, Topology 15 (1976), 111-130. 
D. Notbohm

Mathematisches Institut

Bunssenstr. 3-5

D-37073 Göttingen

Germany

e-mail: notbohm@at.cfgauss.uni-math.gwdg.de

(Received: March 25, 1999) 